\title{
COMPARATIVE REVIEW FOR ROUTING PROTOCOLS IN MOBILE AD-HOC NETWORKS
}

\author{
Hatem S. A. Hamatta, Nabeel I. Zanoon and Rasha M. Al-Tarawneh \\ Department of Applied Sciences, Al-Balqa’ Applied University, Aqaba, Jordan
}

\begin{abstract}
Wireless Mobile Ad-Hoc Networks is one of the attractive research field that growing exponentially in the last decade. it surrounded by much challenges that should be solved the improve establishment of such networks. Failure of wireless link is considered as one of popular challenges faced by Mobile Ad-Hoc Networks (MANETs). As this type of networks does not require any pre-exist hardware. As well as, every node have the ability of roaming where it can be connected to other nodes dynamically. Therefore, the network internal structure will be unpredictably changed frequently according to continuous activities between nodes that simultaneously update network topology in the basis of active ad-hoc nature. This model puts the functionality of routing operation in crucial angle in the area of research under mobile adhoc network field due to highly dynamic nature. Adapting such kernel makes MANET indigence new routing techniques to settle these challenges. Thereafter, tremendous amount of routing protocols proposed to argue with ad-hoc nature. Thus, it is quite difficult to specify which protocols operate efficiently under different mobile ad-hoc scenarios. This paper examines some of the prominent routing protocols that are designed for mobile ad-hoc networks by describing their structures, operations, features and then comparing their various characteristics.
\end{abstract}

\section{KEYWORDS}

MANET, Proactive Routing Protocols, Reactive Routing Protocols, Hybrid Routing Protocols

\section{INTRODUCTION}

During development cycle in the modern technologies, three types of wireless networks became clear; Infra-structured networks, ad-hoc networks and combination of both. The first wireless network is based on bridges known as base stations. Mobiles within these networks need to connect and communicate with one base station which is the nearest to. Thus, according to mobility metric in this kind of networks, each node leaves the range of the base station that it communicates with should be covered with range of another base station. A hand-off process is required between the old base station and new one. In this case the mobile node will be able to keep its communication easily throughout the entire network. Such kind of networks is called Wireless Local Area Networks (WLANs), which is suitable for use in controlled and identified environments (i.e. Offices and universities etc.). In contrast to infra-structure network with base stations which provide coverage for mobile nodes, an ad-hoc network neither have access to base stations nor fixed routers and did not have any controlled environments. These networks can be identified in any environment. Here every node moves unpredictably and dynamically without any support from infra-structured network components that restrict network construction. Therefore, the nodes operate as routers which discover and maintain routes to other nodes in the network. Thus, the network can be constructed any time anywhere (i.e. emergency searches and meetings etc.).

DOI : 10.5121/ijasuc.2016.7202 
This paper reviews different types of routing protocols designed for ad-hoc networks. In the beginning it describes the evolution of ad-hoc networks, and then describes the design goals and the challenges for routing in this kind of network. On the other hand, section 3 describes the operation of each protocol and its characteristics. While the later section shows general comparisons between routing classifications; and finally, conclusion is included in the last section of the article.

\section{EvOLUTION OF AD-Hoc NETWORK}

At the beginning of the 70's, Abramson with his fellow researchers at the University of Hawaii invented ALOHAnet which was the first public demonstration of a wireless packet data network [2]. It utilized single-hop wireless packet switching and multiple access solutions for sharing a single channel. In addition to the need of fixed single-hop wireless network implementation in ALOHAnet as originally it was, the basic idea was compelling and applicable to any environment where access to available resources had to be negotiated among a set of uncoordinated nodes [13], it started operation of providing public demonstration of wireless packet data network in 1971. Also, it triggered widespread interests in different directions of computer communication according to its success and novelty.

In 1972, a new project had been developed; called Packet Radio Network (PRNet) to provide a packet wireless network military application. PRNet was sponsored by the defense advanced research project agency (DARPA) [13], and [9]. PRNET uses a combination of ALOHA with Carrier Sense Multiple Access (CSMA) to access the shared radio channel. It was designated to support self-organized, self-configured, and detect radio connectivity for the dynamic operation of Routing Protocol without any support form fixed infrastructure which proved the feasibility and efficiency of infrastructure-less network and their applications for civilian and military purpose.

The Survivable Radio Network (SURAN) appeared in 1980 as an extended to the work on multihop wireless networks to provide ad-hoc networking with small, low-cost, low-power devices with efficient protocols and improved scalability and survivability [18].

Hence, Mobile Ad-Hoc Network can be defined as a set of multi-hop wireless mobile nodes, which communicates with each other in the case of absence of established infrastructure or any centralized administration. Thus, the main goal of MANET is to improve and extend the mobility of nodes where these nodes must form the network routing infrastructure in ad-hoc environments. The hotspot of MANETs applications is the need of rapid deployment and dynamic configuration, reconfiguration with absent of infrastructure network components (i.e. military battlefields, emergency search, rescue sites and class rooms).

\section{AD-Hoc Mobile Routing SCHEMES}

Since mobile nodes in MANETs are capable of communicating with each other without any existing of networks infrastructure. These nodes are not bounded to any centralized control such as base stations or mobile switching centers. For this reason, the communication sessions can be achieved either through single-hop transmission in case the destination node placed within the transmission range of the source node, or by multi-hop transmission if the packets must relaying through intermediate nodes to reach the destination node in case it placed outside the range of source node [1],[10] and [15]. In MANET, each node behaves not only as a host but also as a router, to form the needed range to give the source node the ability to communicate with the destination node without direct wireless transmission range between them. Thus, in this kind of 
networks each node participates in an ad-hoc routing operation, setting up and maintaining routing information which allows the form of multi-hop range to any other node through the network. So, designing efficient routing protocol in such a network is a challenging problem regarding to its unique characteristics, such as dynamic changing topology, cooperation between nodes, lack of centralized management and monitoring facility, restricted power supply, and scalability. This indicates no stable communication infrastructure [12]. For this reason, routing in ad-hoc network has become a popular and interesting research topic.

Routing is the process of determining a good path thru the network from source node to some arbitrary destination nodes. So, movements of mobile nodes in MANET cause the nodes to move in and out the range from one another. Thus, there is a rapid establishment and breaking of links in the network, forcing the stability of network topology to change dynamically with time. For this reason, traditional routing protocols which are used in wired network cannot be applied directly to wireless and ad-hoc network. Thus, several considerations design issues are defined by the Internet Engineering Task Force (IETF) which is required in routing protocols to be used under mobile ad-hoc network requirements listed as follow [11]:

- Distribution operation

- Demand-based operation

- Proactive operation

- Routing overhead

- Multi-hop routing capability

- Dynamic topology maintenance

- Self-starting and recovery

- Loop prevention

Since the network relies on multi-hop transmissions in its communications, new challenges are derived to determine the multi-hop route over which data packets can be exchanged between source and destination mobile nodes. The most important factor to measure the quality of routing protocols in ad-hoc networks is identified by the ability of adapting the variations of network topology dynamically. According to the initiation time for routing activities, routing protocols for mobile ad-hoc network can be broadly classified into three basic categories: 1) proactive or tabledriven routing protocols, 2) reactive or on-demand routing protocols, and 3) hybrid routing protocols which is combination of both proactive and reactive routing protocols. Figure 1 shows main classifications and some representative examples of routing protocols classes that can be used in mobile ad-hoc network.

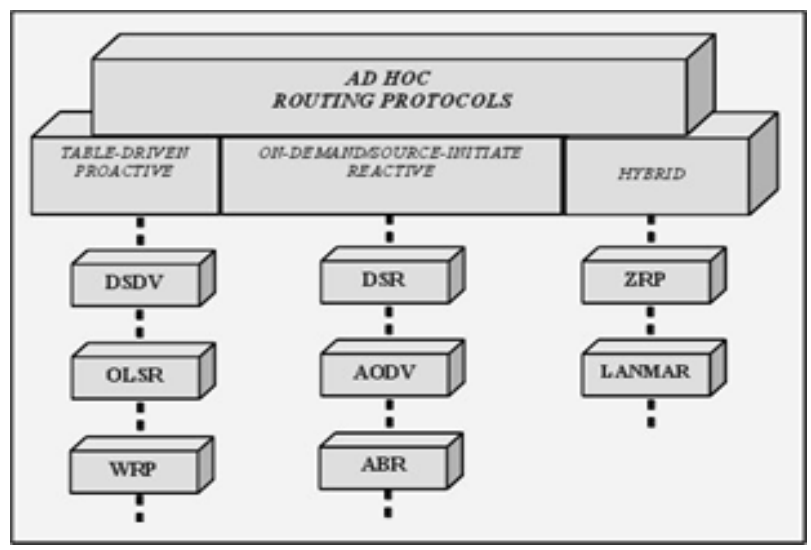

Figure 1. Classification of Ad-Hoc Routing Protocols 


\subsection{Proactive Routing Protocols}

It is also called Table-Driven routing protocols; it attempts to maintain consistent, up-to-date routing information from each node to every other node in the network. Also, such protocols require each node to maintain its routing table in which it keeps tables updated with the acquisition of new information. Thus, table-driven proactive routing protocols respond to changes and updates throughout the network in order to maintain a consistent network view. It has advantages of providing lower latency in data delivery, whilst its main drawback is the wastage of bandwidth in periodically sending update packets even when they are not necessary, such as when there is no link failure, or only a few amounts of routes are required. Some proactive MANET protocols include: DSDV [17], OLSR [6], and WRP [14] are described in the following subsections, where some of their basic characteristics are shown in table 1.

Table 1. Basic Characteristics of Some Proactive Ad-Hoc Routing Protocols.

\begin{tabular}{|l||lll|}
\hline Parameter & DSDV & OLSR & WRP \\
\hline \hline Route Selection Metric & Link State & Multipoint & Shortest Path \\
& & Relay & \\
Routing Uniformity & Uniform & Uniform & Uniform \\
Multiple Route & No & No & No \\
Topology Structure & Flat & Flat & Flat \\
Routing Update Time & Hybrid & Periodic & Hybrid \\
Update Information Metric & Distance Vector & Link State & Distance Vector \\
Beacon & Yes & Yes & Yes \\
Loop Free & Yes & Yes & Yes, but not \\
Critical Nodes & & & consistent \\
No. of Tables & No & MPR & No \\
Control Overhead & 2 & 7 & 4 \\
& High & Low & High \\
\hline
\end{tabular}

\subsubsection{Destination-Sequenced Distance Vector Routing Protocol (DSDV)}

Destination-Sequence Distance Vector Routing Protocol is described by [17] as one of the most important proactive routing protocols that had been developed earlier for mobile ad-hoc networks. Few modifications were applied on the classical Bellman-Ford routing mechanism as specified by RIP [8] to fulfil the needs of stable environment in mobile ad-hoc network. The improvements made to the Bellman-Ford algorithm cover freedom from loops in routing tables. This was implemented by adding a sequence number (originally generated by destination node) with each distance vector entry to indicate the stability and freshness of that entry, thereby avoiding the formation of routing loops.

According to the needs of stable network, routing table updates are periodically transmitted throughout the network in order to give all nodes a consistent view of network layout; figure 2 shows the routing table for node $\mathbf{N} \mathbf{~ i n ~ a d - h o c ~ e n v i r o n m e n t . ~ A c c o r d i n g ~ t o ~ t h a t , ~ h u g e ~ a m o u n t ~ o f ~}$ network traffic will appear. Two types of packets are defined to solve update problems; the first type is known as a full dump packet, which is used infrequently to carry out all available routing information. Whereas, the second one is smaller than full dump packet, it is called incremental packet, which is used to relay only that information which has changed since last full dump. 


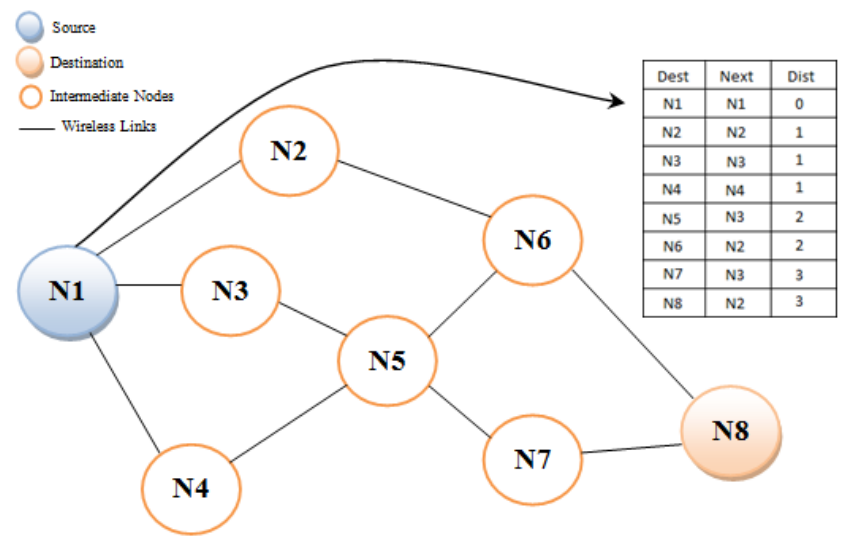

Figure 2. Example of Routing Table in DSDV

The key point of DSDV is the way it handles those types of packets, to keep track of both stale and fresh information that describes the network layout with minimal impact on the network overhead. Thus, there are two routing tables kept at each node. One is used for forwarding packet, while the other is advertised via incremental routing information packets. Accordingly, full dump is responsibility of the first table while the responsibility of the second table is incremental packet.

At each time new route broadcast occurs, the following information are included in broadcasted packet; 1) the address of the destination, 2) number of hops to reach the destination, 3) the destination sequence number, 4) unique broadcast sequence number[17]. The nodes always use the rout labeled with the most recent sequence number. But, if there are two updates with the same sequence number, the route with the smaller metric will be used because it implements the shortest path to the destination. Also, the nodes keep track of the settling time of routes, or the average time that routes to a destination will fluctuate before the route with the best metric is received. DSDV manages the freshness of the routes by delaying the broadcast of a routing update by the length of the settling time; nodes can reduce network traffic and optimize routes by eliminating those broadcasts that would occur if a better route was discovered in the very near future.

\subsubsection{Optimized Link-State Routing Protocol (OLSR)}

OLSR is a proactive table-driven routing protocol designated for mobile ad-hoc networks. It is an optimized version of a conventional link-state routing algorithm in which every node maintains its own information about the network. It is considered as a flat routing protocol, since it does not require any central administration control to handle routing process. It differs from other routing protocols according to its ability to minimize the number of required control messages. Consequently, it gives the network a fresh network topology layout. The key point behind improvements founded in OLSR is achieved by dividing the network into a set of groups each with its specified roles. Groups cooperate with each other to determine the required optimization implemented in OLSR. Thus, OLSR adapt this issue with respect to all possible overhead by defining special group of nodes called Multipoint Relay (MPR), which includes the minimum number of the symmetric "1-hop" neighbours from which it is possible to reach all the symmetrical strict "2-hop" neighbours in order to calculate the optimal MPR set, as shown in figure 3. Any node belongs to MPR set of node $N$ must satisfy the following conditions:

- $\exists X \mid X \in$ Neighbours set of node $\mathrm{N}$. 
- $\forall \mathrm{K}$ in 2-hop neighbourhood of node $\mathrm{N} I \mathrm{~K} \in$ Neighbours set of node $\mathrm{X}$, also must have a bi-directional link to $\mathrm{X}$.

- $\quad$ IMPR(N)I must be the minimal list of neighbours that cover the 2-hop neighbourhood of node $\mathrm{N}$ with bi-directional links.

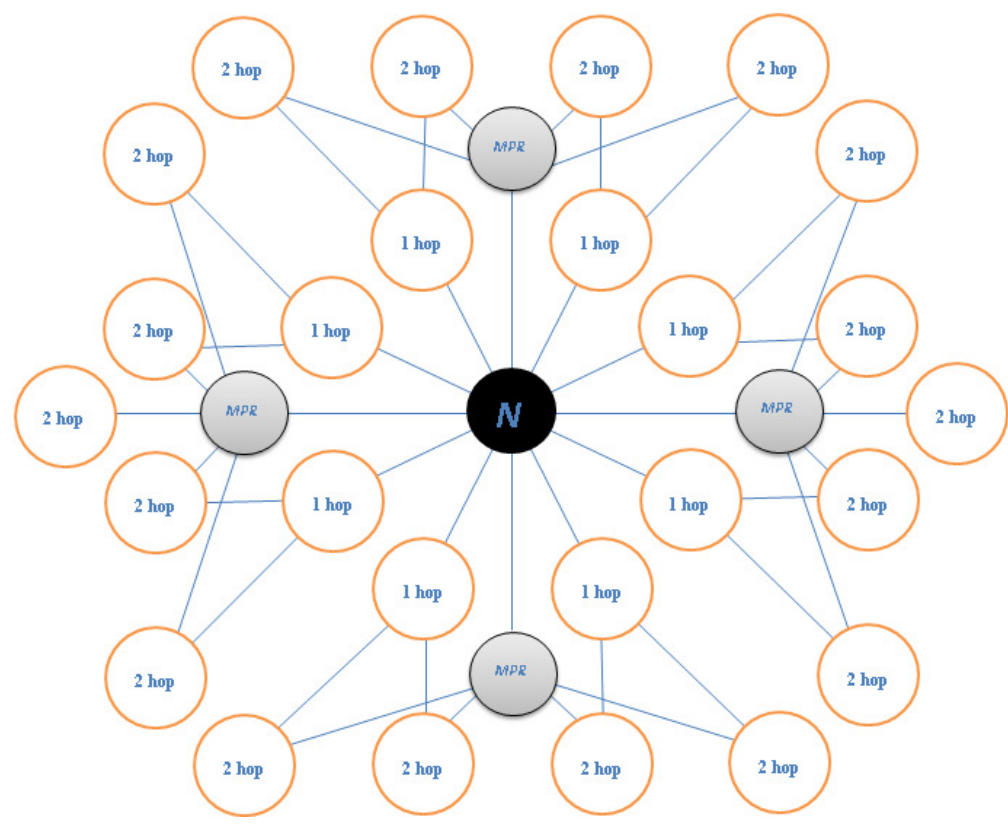

Figure 3. Multipoint Relays

The idea of MPR is to improve flooding process of broadcasts by reducing the number of nodes that occupy the information exchange throughout the network. Many data structures are introduced by OLSR; those must be adapted by each node in the network:

- Link Table.

- Neighbours Table.

- 2-Hop Neighbours Table.

- MPR Table.

- MPR Selector Table.

- Topology Table.

- Routing Table.

Thus, to perform OLSR operations with these various data structures the protocol also defined two special packets structure; HELLO, and Topology Control (TC) messages. Hello message generally serves three important independent tasks: (1) link sensing, (2) neighbour detection, and (3) MPR selection signaling. Since these tasks are based on periodic information exchange within a node neighbourhood and also it serves the common purpose of local topology discovery. Thus, it affects all information that exists in link table, neighbours table, MPR table, and MPR selector table. Link table can be accomplished through periodic of HELLO message and the table hold information about the local links, while neighbours table can be deducted directly from information exists in link table about the entire symmetric 1-hop neighbourhood to perform neighbour detection. Thus, it is considered as a part of link sensing. On the other hand, MPRs of a given node (say $\mathrm{N}$ ) are declared according to subsequent HELLOs which transmitted from given node, consequently, this information will reach the multipoint relays themselves. So, any node can belong to $\mathrm{MPR}(\mathrm{N})$ if and only if: 
International Journal of Ad hoc, Sensor \& Ubiquitous Computing (IJASUC) Vol.7, No.2, April 2016

- It belongs to symmetric 1-hop neighbours of $\mathrm{N}$ (is considered as neighbour of $\mathrm{N}$ ).

- It enables node $\mathrm{N}$ to reach 2-hop neighbours.

- It has the willingness to be MPR.

Consequently, MPR(N) are also do the same process of broadcast HELLO message periodically for its 1-hop neighbours, node $\mathrm{N}$ will consider that those nodes set themselves as MPR(N) and accordingly, it will update its MPR SELECTOR table in which it puts the addresses of its 1-hop neighbours nodes which has been selected as a multipoint relays along with the corresponding MPR sequence number of that neighbour node. All previous data structures are defined in OLSR to be the base infrastructure of transmission of TC. It is considered as the only way the node can exchange its own links in the network through its MPR sets, the node must send at least link of its MPR selector set. The sequence number associated with each MPR is also attached to the list. The freshness of the messages is recognized using higher sequence number. Also, it is used to avoid loops of the messages by discarding TC packets with same or smaller sequence number. The advantage of TC is helping each node to build its own topology table and give stable view of the network layout since the node continuously updates its topology table according to it.

The main difference detected between OLSR and other routing protocols is; its ability to give each node the required infrastructure and needed data structure to maintain its routing table locally, which gives the ability to route the packets towards other destinations in the network. The information available in neighbours and topology tables are the base of routing table in OLSR, each node re-calculates its routing table if any changes occur in any of link table, neighbours table, 2-hop neighbours table, or topology table. It maintains its routing information accordingly with respect to finding the shortest path for all routes that will be inserted in routing table. Although, routing table re-calculation required a lot of information about the network. In OLSR it does not trigger any packet to be transmitted neither in the entire network, nor in the 1-hop neighbourhood. Accordingly, it is clear that OLSR protocol is well suited to work on a dense network where most of the communication is concentrated between large numbers of nodes.

\subsubsection{Wireless Routing Protocol (WRP)}

Another type of proactive (table-driven) distance vector routing protocols is WRP that is used in multi-hop packet-radio network. It follows the shortest-path routing techniques. Typically, it is based on Distributed Bellman-Ford algorithm (DBF) [8]. In this protocol, all routing nodes know the length of the shortest path through its neighbours to every destination node in the network. It proposed a Path-Finding-Algorithm (PFA), to deal with temporary looping situation. Also, the protocol limits routing table updates to include only that entries affected by network changes.

The main updating function in WRP is performed only between neighbourhoods. It is designed to run on the top of the medium access control protocol of a wireless network. Also, it ensures reliable transmission of update messages as it adapts the mean of re-transmissions. This can be achieved by sending a positive acknowledgment (ACK) after receiving an updating message free of errors. This ACK can be considered as an assertion to neighbourhood between nodes. The protocol maintains three tables and one list. First table is a distance table which implements required information toward all destinations in the network, the number of hops is considered as a distance to a particular destination, next hop to reach destination must be specified within each entry. Second table, the routing table gives full specification vector for each known destination in the network as shown in figure 4. This specification is (Destination Identifier, Distance to the Destination, Shortest Path, and specified Tag which identifies the status of that entry if it corresponds to simple path or loop). Third table is link cost table. It gives the cost of relaying information through neighbours. Also, status of links with neighbours is attached. At the end, the 
message re-transmission list (MRL) specifies re-transmission entries. It gives each node the ability to know which updates have to be re-transmission.

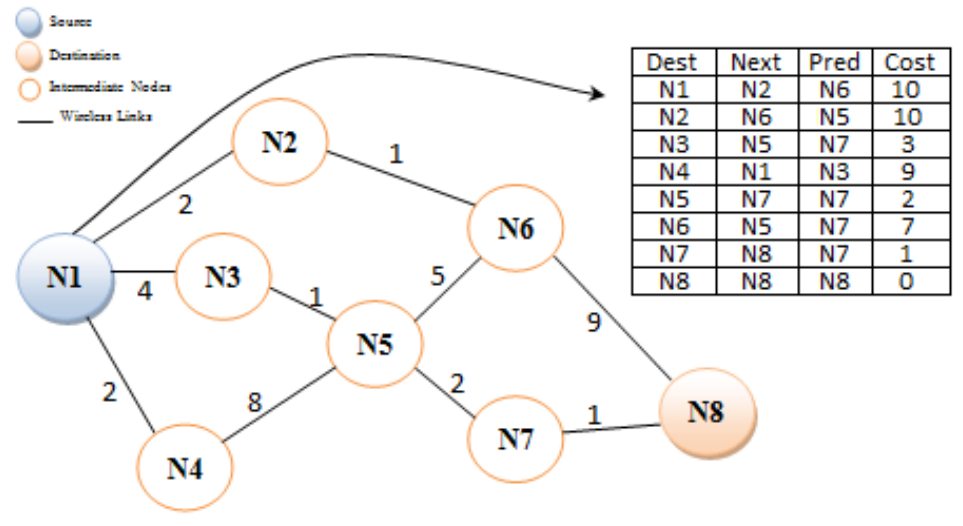

Figure 4. Routing Table in WRP

WRP operates smoothly in information exchange among nodes. This means, it permits propagation only between neighbours. Accordingly, the first update should ask all neighbours to send an ACK. Consequently, when there is no updates, null update message called HELLO message will be sent to the neighbours, where the time interval between two successive messages is "HELLO Interval". Each node in the network can decide to update its routing table after a new update has been received from its neighbours or any changes in the link status with its neighbours received. Accordingly, in case of receiving updates, they are processed in order. After that, the node should generate a new update message toward its neighbours.

\subsubsection{Summary of Proactive (Table-Driven) Routing Protocols}

Updating procedure in MANETs consumes significant amount of network bandwidth in most of table-driven routing protocols. Accordingly, global routing does not scale very well [1]. The reason is, each node should update its routing information for all nodes belong to the same network. This process should be repeated periodically to keep track to the last network topology layout. Thus, the attractive difference between these protocols is the way they handle the view of network topology. Thus, table 2 maintains the main advantages and disadvantages for the examined proactive routing protocols.

Table 2. Basic Characteristics of Some Proactive Ad-Hoc Routing Protocols

\begin{tabular}{|c|c|c|}
\hline Protocol & Advantages & Disadvantages \\
\hline DSDV & $\begin{array}{l}\text { (1) Loop free. } \\
\text { (2) Simple computationally efficient. }\end{array}$ & $\begin{array}{l}\text { (1) Excessive communication overhead. } \\
\text { (2) Slow convergence. } \\
\text { (3) Tendency to create routing loops in } \\
\text { large networks overhead. }\end{array}$ \\
\hline OLSR & $\begin{array}{l}\text { (1) Reduce size of update message } \\
\text { and number of transmissions than } \\
\text { a Pure link state routing protocol }\end{array}$ & $\begin{array}{l}\text { (1) It requires information of both 1- hop } \\
\text { and 2- hop neighbors }\end{array}$ \\
\hline WRP & $\begin{array}{l}\text { (1) Loop free. } \\
\text { (2) Lower worst case time complexity than } \\
\text { DSDV. }\end{array}$ & $\begin{array}{l}\text { (1) Prevent sleep mode. } \\
\text { (2) A large amount of memory and } \\
\text { periodic hello message consumes power } \\
\text { and bandwidth. }\end{array}$ \\
\hline
\end{tabular}




\subsection{Reactive Routing Protocols}

Reactive routing protocols are designed to minimize routing overhead. It is also called, sourceinitiated on-demand routing. Instead of tracking all changes in the network topology to determine the shortest path to all destinations, on-demand protocols trigger this process only when necessary. Mainly, these protocols perform two operations. First, ROUTING DISCOVERY which is triggered when a node requires route to particular destination. Routing discovery process is performed by source node (source-initiated). Second, ROUTING MAINTENENACE; Once a route is established, it is maintained by routing maintenance until either the destination becomes inaccessible along every path from the source or until the needs for route is finished.

The main advantage of on-demand nature operations is that it usually has lower average of routing overhead if it is compared with proactive routing protocols. Due to that, reactive routing protocols receive more attention than proactive routing protocols. Since they are more stable for dynamic environments in MANET. Various parameters were used for reactive routing protocol improvement to come up with the proactive technique circumstances shown in table 3 . Accordingly, these reactive on-demand protocols include: DSR [7], AODV [16], and ABR [19] which described in the following subsections.

Table 3. Basic Characteristics of Some Reactive Ad-Hoc Routing Protocols

\begin{tabular}{|l||lll|}
\hline Parameter & DSR & AODV & ABR \\
\hline \hline Route Selection Metric & Shortest Path & Shortest Path & Signal Strength or \\
& Updated Path & Updated Path & $\begin{array}{l}\text { Associativity And } \\
\text { Shortest Path }\end{array}$ \\
Routing Uniformity & Uniform & Uniform & Uniform \\
$\begin{array}{l}\text { Multiple Route } \\
\text { Topology Structure }\end{array}$ & Yes & No & Yes \\
Routing Update Time & Flat & Flat & Flat \\
& Event Driven & Event Driven & Event Driven \\
Update Information & Route Error & Route Error & /Periodically \\
Metric & & & Route Error \\
Beacon & Yes & No & \\
Loop Free & Yes & Yes & Yes \\
Critical Nodes & No & No & Yes \\
Route Reconfiguration & Erase Route & Erase Route and & No \\
& and Inform & Inform Source & Localized \\
& Source & & Broadcast Query \\
& & & Until Find A Route \\
Control Overhead & Low & Low & Low \\
\hline
\end{tabular}

\subsubsection{Dynamic Source Routing Protocol (DSR)}

The Dynamic Source Routing protocol is one of reactive on demand routing protocols that is based on distance vector technique. This protocol adopts the concept of source routing and derived routing techniques in which the sender explicitly lists the complete sequence of nodes required to reach destination in the packets header. This concept identifies each forwarding "hop" using the address of the next node to which the transmitted packet will be advanced on its way until it reaches its destination. DSR is explicitly initiated for wireless environments of an adhoc network. It dynamically determines the routes needed to reach destination. Thereby, there is no periodic advertisement in the protocol while it reduces the network overhead.

The basic operation of DSR protocol starts at sender node as it constructs a source routing in packet's header. After that, the sender transmits the packet to the first hop listed in the source 
route of the received REPLY packets. The first hop node check whether it is the final destination of the packet's or not, and it simply transmits the packet to the next hop listed in the source route in the packet's header until the packet reaches its final destination.

To allow all nodes to participate in the ad-hoc routing, each one maintains its source routes in its route cache. Accordingly, for any node willing to send a packet to another node, it first checks its route cache for a source route to the destination. In case route is found, the sender uses it to propagate the packet directly to the destination. These routes are discovered on demand using process called Route Discovery as shown in figure 5.

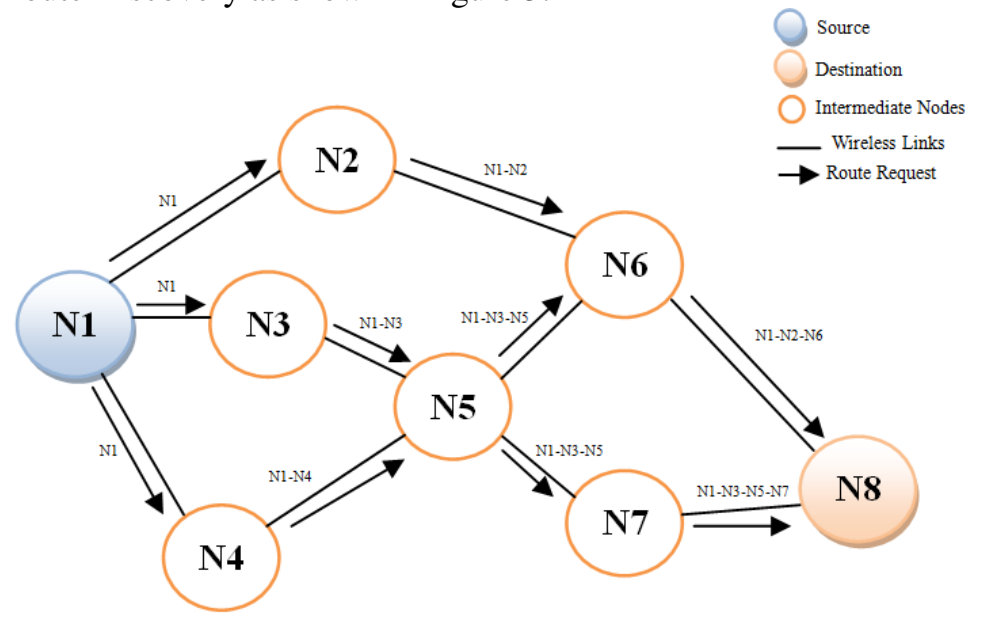

Figure 5. Route Discovery in DSR

In route discovery operation, Route Request Packet is initiated for seeking a route to specified destination. This packet contains information about the source and destination. According to that, any node that receives a Route Request Packet first checks its route cache for any available information about its listed destinations as shown in figure 5. Subsequently, the node adds its identity to the header of Route Request Packet and then broadcasts it. When information is available or Route Request Packet reach destination a Route Reply Packet will be sent back to the source in reverse direction as illustrated in figure 6 . This is simply done by copying the sequence of nodes identities collected from Route Request Packet.

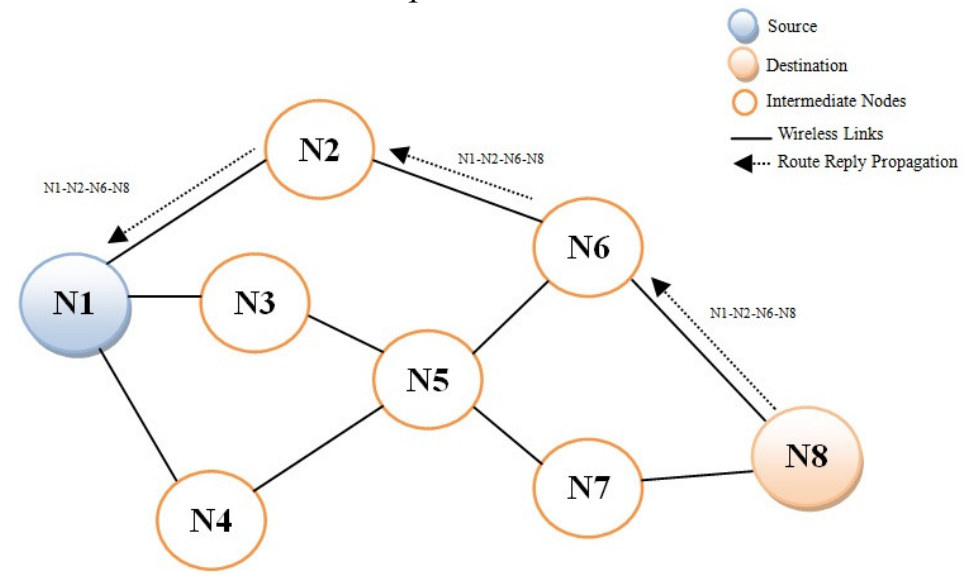

Figure 6. Route Reply Propagation in DSR 
The Route Reply Packet contains the entire route to the destination which is recorded in the route cache of the source node. On the case of link failure, the node that had detected the route break sends ERROR Packet back to the source. In this case, all nodes in this sub-path including the source will delete all existing information about this route from their route cache and a fresh route discovery process will be initiated if a route is still needed.

\subsubsection{Ad-Hoc On-demand Distance Vector Routing Protocol (AODV)}

This protocol is considered as an improvement of the Destination-Sequenced Distance Vector (DSDV). Unlike DSDV, AODV minimizes the number of required broadcasts as it creates and maintains routes only when they are needed. Thus, it limits the time used for route acquisition. The protocol uses traditional routing tables and only one entry per each destination. Also, it utilizes sequence number to examine the freshness of the stored routes in routing table. Also, it ensures loops free routing. Thus, each routing table entry is "expired" after a certain predetermined threshold of time.

In contrast to DSR, AODV uses a different mechanism to create routes, when a source node desires to send a message to another node whereas it does not already have a valid route to that destination, it applies a path discovery operation to find out fresh valid path to that destination. Accordingly, it broadcasts a route request packet to its neighbours, all nodes presented (between source and destination) are occupied as intermediate nodes. Route Discovery operation is completed either by any intermediate node which has a fresh enough valid route to the requested destination or by the destination itself.

The path freshness is determined by the destination sequence number, which is compared with available information in an intermediate node for a requested path. At this moment, if the intermediate node does not have any information about that destination or it has entry for that destination in its routing table with destination sequence number less than the one in route request packet, it proceeds forwarding routing request to its neighbours until it reaches any node which has valid route for that destination or it reaches destination itself. Route Discovery can be finalized only by destination or intermediate node if its recorded entry sequence number is greater than the one in Route Request. Consequently, it establishes unicast Route Reply Packet backward along the reversed path from which it first receives the Route Request. Accordingly, every node belongs to this path sets up its forwarding route entries in their routing tables which refer to the node from which Route Reply came as shown in figure 7 and figure 8 respectively. Thus, valid path will be achieved for forwarding process.

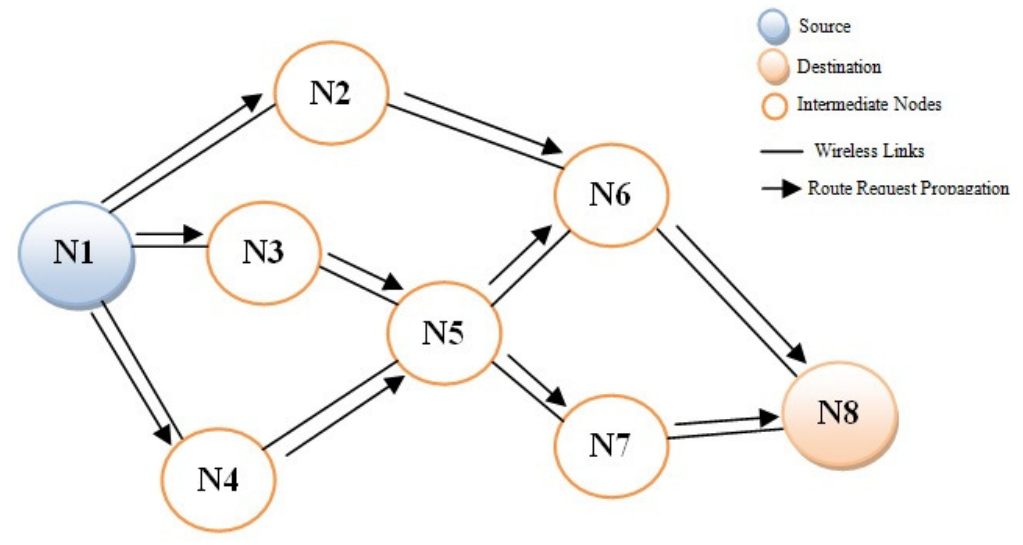

Figure 7. Route Request in AODV 


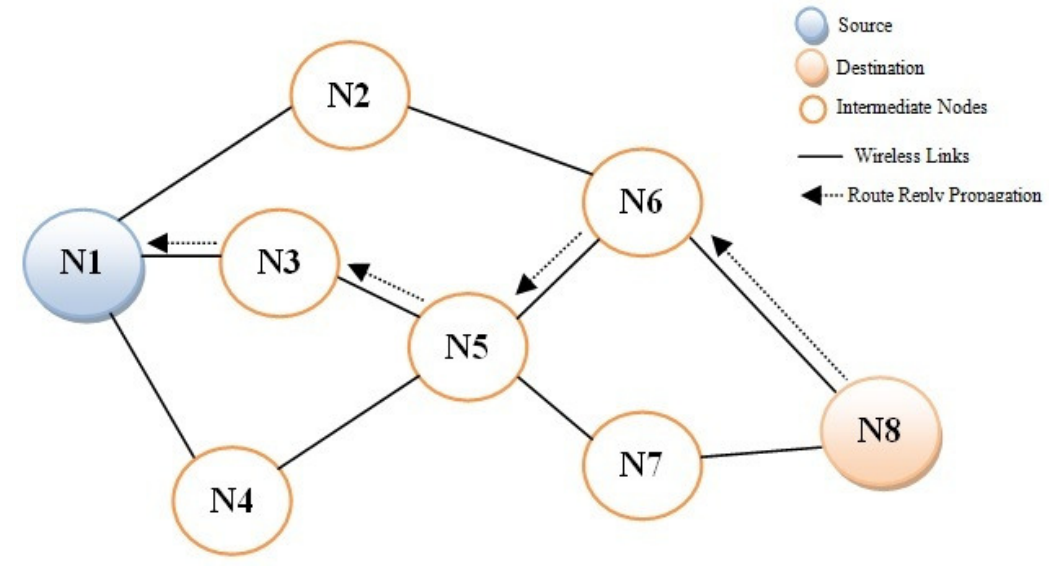

Figure 8. Route Reply in AODV

Another operation is very important in AODV. That is, Routing Maintenance Operation, it provides route recovery in case of path breaks due to link failure or node movements as shown in figure 9. This failure cannot be handled locally. So, a special unsolicited route reply will be sent to source node that initiates this broken path with fresh sequence number and hop count is set to $\infty$ [16]. This packet instantiated by those nodes where its next hop become unreachable, the special route reply packet notifies all nodes in the path about the link breaks. Consequently, all of these nodes reply that packet to their active neighbours and so on. At the time of reaching the packet to the source, it can restart route discovery in case it still requires a path to that destination.

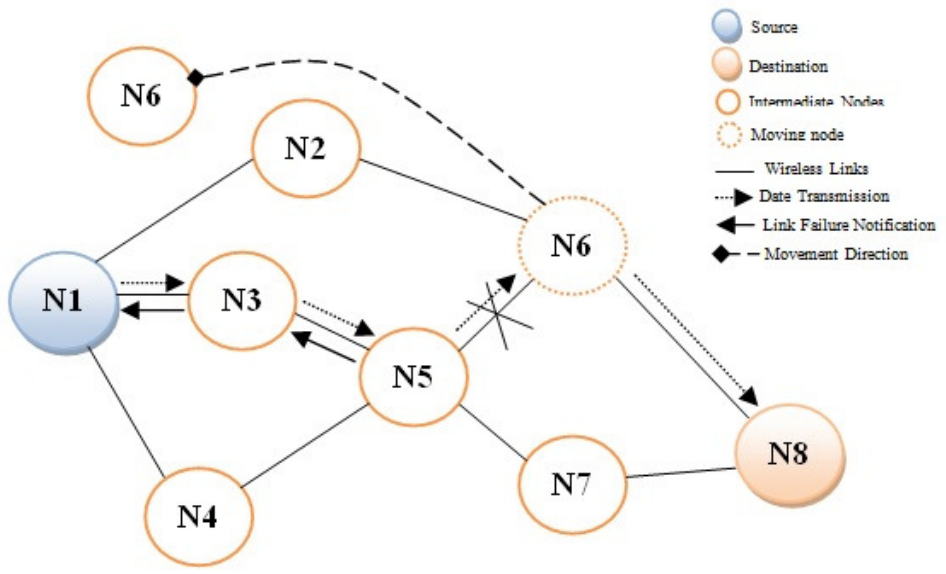

Figure 9. Routing Maintenance in AODV

\subsubsection{Associativity-Based Routing Protocol (ABR)}

$\mathrm{ABR}$ is one of reactive source-initiated routing protocols that employ an associativity-based routing scheme where a route is selected according to the period of stability [19]. This interval of time will be the metric which helps the destination to choose one from the available routes as the best route for specific connection. The metric was defined on the basis of the movement time of particular node as an unstable period. This process can be defined using associativity "ticks" which is defined by Route Selection Rule. According to this rule, three different cases were specified to gain with the best route:

- Case 1: choose the highest associativity "ticks" route. 
International Journal of Ad hoc, Sensor \& Ubiquitous Computing (IJASUC) Vol.7, No.2, April 2016

- Case 2: if some routes have same highest associativity "ticks", then the one with the minimum hop-count will be chosen.

- Case 3: if some routes have same highest associativity "ticks" and same minimum hopcount then any one of these routes will be arbitrary selected.

The route selection metric is executed as a consequence to first phase of ABR routing protocol. Accordingly, two other phases are required to completely identify ABR routing protocol. These phases are:

\section{Route Discovery Phase:}

It is considered as the first executed phase of ABR routing protocol. It computes the approximation of the data throughput associated with any selected route. This operation depends on the availability of knowledge about associative "ticks" for neighbours in the route. Also, the relaying load of nodes supporting route should be known.

This phase initially, assumes there is no information about the designated Destination in all nodes in the network except destination's neighbours. In case any node needs a route to the Destination, it broadcasts a special message called Broadcast Query (BQ) message. The BQ message will be flooded throughout the entire network until it reaches any node that has a valid route to the destination. To reduce the network overhead BQ message attached with a uniquely identified sequence number where the amount of time BQ message transmitting should not exceed once. Consequently, all intermediate nodes examine the uniqueness of the BQ message whether it accessed before or not. According to that, if the node is not the destination, the node will append its address in $\mathrm{BQ}$ message and pass it to its neighbours. Information like relaying load, propagation delay of the link and the hop-count will be appended in BQ message for all intermediate nodes between source and destination.

At the Destination side, ABR Route Selection Algorithm will be executed at an appropriate time after receiving the first $\mathrm{BQ}$ message to select the best route from all possible known routes. Accordingly, the Destination sends a REPLY packet through the selection route. All intermediate nodes listed in reply packet update their routing information as a valid route. Otherwise the node will not be able to forward any packet to that destination even if it hears the transmission. In this case, no duplicate packets will reach destination.

Eventually, the source will send another BQ packet in case it never receives REPLY from destination after predefined BQ-timeout. Accordingly, all intermediate nodes will consider the new $B Q$ packet according to its unique sequence number of the same destination.

\section{Route Re-Construction Phase:}

This phase is considered as a route maintenance phase. It performs a set of operations implemented in ABR routing protocol, which are:

- Partial Route Discovery.

- Invalid Route Erasure.

- Valid Route Update.

- New Route Discovery. 
International Journal of Ad hoc, Sensor \& Ubiquitous Computing (IJASUC) Vol.7, No.2, April 2016

In this protocol, the chosen route is more likely the one with long-lived that based on the property of associativity. Accordingly, any unexpected moves should be recorded. The protocol will try to locate alternative valid route quickly without re-broadcasting query message, unless necessary.

\section{Route Deletion Phase:}

It is counted as the last step in ABR routing protocol as it starts when a particular route is no longer needed. To do this, the source initiates a route delete broadcast; accordingly, all intermediate nodes update their routing entries.

\subsubsection{Summary of Reactive (on-Demand) Routing Protocols}

As on-demand routing protocol designed to minimize the high overheads of maintaining routing information in proactive routing protocols, it applies routing discovery only for active and required routes. Thus, the node can predict its connectivity to other nodes only on demand to those nodes. As in proactive, all nodes participating on finding path when source requests that path for designated destination. Reactive routing reduces the impact of network topology consistency, since this is not required. This was the core idea of reactive routing technique. But, latency time will be increase according to the route discovery process which increases the time for delivering data to the destination. List of advantages and disadvantages of examined reactive on demand routing protocol as shown in table 4.

Table 4. Basic Characteristics of Some Proactive Ad-Hoc Routing Protocols

\begin{tabular}{|c|c|c|}
\hline Protocol & Advantages & Disadvantages \\
\hline DSR & $\begin{array}{l}\text { (1) Multiple route } \\
\text { (2) Promiscuous overhearing } \\
\text { (3) No periodic hello message and fast } \\
\text { recover }\end{array}$ & $\begin{array}{l}\text { (1) Scalability problems due to the } \\
\text { nature of source routing } \\
\text { (2) Large delay and large networks } \\
\text { overhead }\end{array}$ \\
\hline AODV & $\begin{array}{l}\text { (1) Adaptable to highly dynamic topologies } \\
\text { (2)Uses bandwidth efficiently } \\
\text { (3)Loop free }\end{array}$ & $\begin{array}{l}\text { (1) Scalability problems } \\
\text { (2) Large delay }\end{array}$ \\
\hline ABR & $\begin{array}{l}\text { (1) Route stability } \\
\text { (2)Avoid packet duplicates } \\
\text { (3) No route reconstruction }\end{array}$ & $\begin{array}{l}\text { (1) Operation complexity } \\
\text { (2) Communication complexity } \\
\text { (3) Scalability problems }\end{array}$ \\
\hline
\end{tabular}

\subsection{Hybrid Routing Protocols}

New generation of Routing protocols are generated to be adapted in mobile ad-hoc networks. These protocols are come up with better balance between adaptability for various network circumstances, conditions, and routing overhead. These protocols adapt the nature of reactive and proactive routing techniques, where each will be applied under different conditions and regions. It increases network scalability, since this improvement is achieved by taking the benefits of dividing the network into zones or clusters and applying proactive routing concepts within each zone or cluster, where reactive routing techniques are applied across different zones or clusters. Thus, it takes the advantages of both pro- and re- active routing techniques to formulate new routing protocol with better performance paradigm. Some of hybrid routing protocols are described in the following subsection such as Zone Routing Protocol [5] and Landmark Ad-Hoc Routing Protocol [3] and [4]. Also, a list of protocols parameters is shown in table 5. 
Table 5. Basic Characteristics of Some Hybrid Ad-hoc Routing Protocols

\begin{tabular}{|l||ll|}
\hline Parameter & ZRP & LANMAR \\
\hline \hline Route Selection Metric & Shortest Path & Shortest Path or \\
& & Quite Close to Shortest \\
Routing Uniformity & Nonuniform & Path \\
Multiple Route & No & Nonuniform \\
Topology Structure & Hierarchical & Yes \\
Routing Update Time & Hybrid & Hierarchical \\
Update Information Metric & Hybrid & Hybrid \\
Beacon & Yes & Hybrid \\
Loop Free & Yes & Yes \\
Critical Nodes & No & Yes \\
Control Overhead & Medium & Landmark \\
\hline
\end{tabular}

\subsubsection{Zone Routing Protocol (ZRP)}

Due to the scalability required for mobile ad-hoc network, Zone Routing Protocol (ZRP) was the first hybrid routing protocol which reduces the control overhead by applying proactive routing protocols and decreases the latency that appears from routing discovery in reactive routing protocols. So, it applies both proactive and reactive ad-hoc routing components.

ZRP was first introduced by Hass in 1997[5]; the key value of this protocol is the ability to divide all networks members into a set of groups called zones. Each zone limited each node with k-hops to reach the zone boundaries. To deploy this concept, ZRP found two types of protocols. First, IntrA-zone Routing Protocol (IARP), it allows all nodes to update and maintain their routing tables to contain consistent routes for all other nodes within the same zone. IARP was constructed under the basis of proactive routing techniques. On the other hand, ZRP built IntEr-zone Routing Protocol (IERP) according to reactive routing concepts to be used between routing zones. Accordingly, for any required route regarding a specified destination located in local zone, source can establish its route from its routing table that cached pro-actively by IARP. Then, the packet can be delivered immediately since both source and destination belong to the same zone. But for other routes beyond local zone, the source broadcasts a route request to those nodes located at the zone border which are called peripheral nodes to communicate with other zone. Accordingly, these peripherals check its local zone whether the requested node is a member of their local zone or not. Consequently, peripherals will forward the request packet to the destination boarder nodes, which will send a route reply on the reverse path back to the source with the full path from source to the required destination. The total overhead of flooding such as path discovery is minimized since it is based on a corporation between peripherals only.

\subsubsection{Landmark Ad-Hoc Routing Protocol (LANMAR)}

This protocol implements the adaptation of fixed wide area network. According to landmark paradigm, the protocol does not require any predefined hierarchal addressing scheme; moreover, it extracts the basis of landmark and modifies it to be applicable for ad-hoc environment. It adapts the concept of landmark that handles group mobility. This scheme solves both mobility and scalability problem. Also, the protocol uses routing scheme that implements some modification on FSR. Since FSR allows each node to have information about the entire network. While LANMAR gives the ability to keep information only about the nodes within the scope of landmark nodes, where these landmarks are elected dynamically.

LANMAR maintains routing information within the group according to proactive link state routing protocols. Also, a distance vector algorithm is used to maintain routing information 
amongst all landmarks. So, every node has a full view about topology of its group where distances of routing vector information to all landmarks are known.

Accordingly, nodes are willing to send a packet for specified destination placed within the same group, the packet will deliver immediately to that destination, since the routing operation based on the accurate routing information available in sender routing tables. Otherwise, the node will pass the packet towards the landmark that corresponds to the destination group, where it may route the packet to the destination directly.

The main advantage of LANMAR protocol is the ability to reduce the overall control overhead through the trimming process of local routing tables. On the other hand, the overhead is totally minimized by the concepts of summarizing routing information to remote groups of nodes.

\subsection{Proactive vs. Reactive vs. Hybrid Routing}

The trade-offs between ad-hoc routing strategies are quite complex. Table 6 shows different parameters that can be taken into account while classifying ad-hoc routing protocols as mentioned in figure 1. Accordingly, to identify which approach is better depends on many factors, such as the size of the network, the mobility, the data traffic and so on. Most of proactive protocols attempt to maintain routes to all possible destinations, whether they are needed or not. Thus, it is continuously propagates and maintains routing information.

In contrast, reactive protocols initiate route discovery on the demand of data traffic. The only routes required to those desired destinations. This routing approach can reduce routing overhead when a network is almost static and the active traffic is not heavy. However, the source node has to wait for routing discovery process to discover valid fresh route to the designated destination, accordingly, increasing the response time.

The hybrid routing approach can adjust its routing strategies that adapt both proactive and reactive techniques according to a network's characteristics and thus provides an attractive method for routing in MANETs. However, a network's characteristics, such as the mobility pattern and the traffic pattern, can be expected to be dynamic.

Table 6. Main Classification of Ad-Hoc Routing Protocol

\begin{tabular}{|c|c|c|c|}
\hline Parameter & Proactive & Reactive & Hybrid \\
\hline Network Architecture & Flat/Hierarchical & Flat & Flat/Hierarchal \\
\hline Topology Distribution & Periodical & When needed & Both \\
\hline Distribution Type & Flooding & Only from Src to Dest & Both \\
\hline Route Availability & Instantaneously & On-demand & $\begin{array}{l}\text { Based on destination } \\
\text { location }\end{array}$ \\
\hline Route Latency & Comprehensive & Available on demand & Both \\
\hline $\begin{array}{l}\text { Communication } \\
\text { Overhead }\end{array}$ & High & Low & Medium \\
\hline $\begin{array}{l}\text { Amount of Local } \\
\text { Information }\end{array}$ & Large & Low & $\begin{array}{l}\text { Based on the node role } \\
\text { and cluster size }\end{array}$ \\
\hline
\end{tabular}

\section{CONCLUSION}

According to the characteristics of mobile ad-hoc networks, set of circumstances should be taken into account when mobile ad-hoc network is required. These networks are based on specific needs which specify their requirements. Network size, structure, movement speed, availability of resources and much more, specify the network requirements and also, the type of ad-hoc that will 
be built. Thus, the protocol uses play significant role and has high impact on the network operations. Since it adjusts the overall networking operations under these circumstances. Accordingly, this paper examines main categories of routing protocols Pro-active, Re-active, and Hybrid routing protocols (Figure 1) that can be applied on MANETs. According to performance metric and those circumstances, implementing such kind of protocols is not easy but it should be simple as much as possible.

Since, all categories focus on the concept of reducing the control overheads. Reactive on-demand protocols perform more efficiently than proactive table-driven protocols since it is not required to have a consistent view of the network according to its rapid changes, while keeping this view without any need will exhaust network bandwidth as well as network resources. So it is beneficially to reduce control overhead by applying reactive routing techniques. But, according to scalability issues, the reactive protocols do not work well. This opens the door for applying hybrid routing protocols which is compromising between proactive and reactive techniques. This category is suitable for large network where it takes its advantages from its ability to apply hierarchal structure for ad-hoc environment. It simplifies routing management by taking kind of adjustment between reactive and proactive routing techniques.

\section{REFERENCES}

[1] Abolhasan, M., T. Wysocki, and E. Dutkiewicz (2004). A review of routing protocols for mobile ad hoc networks. Ad Hoc Networks 2 (1), 1 - 22. DOI : "10.1016/S1570-8705(03)00043-X".

[2] Abramson, N. (1970). The aloha system: another alternative for computer communications. In Proceedings of the November 17-19, 1970, fall joint computer conference, AFIPS '70 (Fall), New York, NY, USA, pp. 281-285. ACM. DOI : “10.1145/1478462.1478502”.

[3] Gerla, M., X. Hong, and G. Pei (2000). Landmark routing for large ad hoc wire-less networks. In Global Telecommunications Conference, 2000. GLOBECOM '00. IEEE, Volume 3, pp. 1702-1706 vol.3. DOI:"10.1109/GLOCOM.2000.891927".

[4] Guangyu, P., M. Geria, and X. Hong (2000). Lanmar: landmark routing for large scale wireless ad hoc networks with group mobility. In Mobile and Ad Hoc Networking and Computing, 2000. MobiHOC. 2000 First Annual Work-shop on, pp. 11-18. DOI:"10.1109/MOBHOC.2000.869208".

[5] Hass, Z. (1997). A new routing protocol for the reconfigurable wireless networks. In Universal Personal Communications Record, 1997. Conference Record., 1997 IEEE 6th International Conference on, Volume 2, pp. 562-566 vol.2. DOI:"10.1109/ICUPC.1997.627227”.

[6] Jacquet, P., P. Muhlethaler, T. Clausen, A. Laouiti, A. Qayyum, and L. Viennot (2001). Optimized link state routing protocol for ad hoc networks. In Multi Topic Conference, 2001. IEEE INMIC 2001. Technology for the 21st Century. Proceedings. IEEE International, pp. 62-68. DOI:"10.1109/ INMIC. 2001.995315".

[7] Johnson, D. B. and D. A. Maltz (1996). Dynamic source routing in ad hoc wireless networks. In Mobile Computing, pp. 153-181. Kluwer Academic Pub-lishers. DOI:”10.1007/978-0-585-29603$6 \_5 "$.

[8] Jr., L. R. Ford. and D. R. Fulkerson (1962). Flows in Networks. Princeton University Press. ISBN: 9780691146676

[9] Jubin, J. and J. Tornow (1987). The darpa packet radio network protocols. Proceedings of the IEEE 75 (1), 21-32. DOI:"10.1109/PROC.1987.13702". 
International Journal of Ad hoc, Sensor \& Ubiquitous Computing (IJASUC) Vol.7, No.2, April 2016

[10] Latiff , L. and N. Fisal (2003). Routing protocols in wireless mobile ad hoc network - a review. In Communications, 2003. APCC 2003. The 9th Asia-Pacific Conference on, Volume 2, pp. 600-604 Vol.2. DOI: "10.1109/APCC.2003.1274428".

[11] Macker, J. P. and M. S. Corson (1998, January). Mobile ad hoc networking and the ietf. SIGMOBILE Mob. Comput. Commun. Rev. 2 (1), 9-14. DOI: “10.1145/584007.584015.

[12] Mohapatra, P. and S. V. Krishnamurthy (2005). Ad Hoc Networks Technologies and Protocols. United States of America: Springer US. ISBN: 978-0-387-22689-7.

[13] Murthy, C. S. R. and B. Manoj (2004). Ad Hoc Wireless Networks: Architectures and Protocols. Upper Saddle River, NJ, USA: Prentice Hall PTR. ISBN: 013147023X.

[14] Murthy, S. and J. J. Garcia-Luna-Aceves (1996, October). An efficient routing protocol for wireless networks. Mob. Netw. Appl. 1 (2), 183-197. DOI : "10.1007/BF01193336".

[15] Perkins, C. (2006). Ad hoc networking in the ietf. In Broadband Convergence Networks, 2006. BcN 2006. The 1st International Workshop on, pp. 1-35. DOI: “10.1109/BCN.2006.1662294”.

[16] Perkins, C. and E. Royer (1999). Ad-hoc on-demand distance vector routing. In Mobile Computing Systems and Applications, 1999. Proceedings. WMCSA '99. Second IEEE Workshop on, pp. 90-100. DOI: “10.1109/MCSA.1999.749281”.

[17] Perkins, C. E. and P. Bhagwat (1994). Highly dynamic destination-sequenced distance-vector routing (dsdv) for mobile computers. pp. 234-244.

[18] Szkutak, P. M. (1985). Design of a survivable packet radio network control algorithm. In Military Communications Conference, 1985. MILCOM 1985. IEEE, Volume 3, pp. 713-717. DOI: “10.1109/MILCOM.1985.4795130:.

[19] Toh, C.-K. (1996). A novel distributed routing protocol to support ad-hoc mobile computing. In Computers and Communications, 1996., Conference Proceedings of the 1996 IEEE Fifteenth Annual International Phoenix Conference on, pp. 480-486. DOI: “10.1109/PCCC.1996.493675”.

\section{AUTHOR}

Dr. Hatem S. A. Hamatta received his B.Sc. degree in Computer Science from Yarmouk University, Irbid, Jordan, in 2003, the M.Sc. degree in Computer Science from University of Jordan, Amman, Jordan, in 2006. He is currently pursuing his research work of $\mathrm{PhD}$ Degree in Wireless Network Security from Aligarh Muslim University, Aligarh, India. Since 2006, he has been with the faculty of the Department of Applied Sciences at Al-Balqa Applied University/Aqaba University College, Aqaba, Jordan; where he is currently Assistant

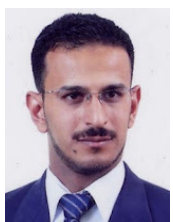
Professor and Dean Assistant for Development and Quality Assurance. His research interests include Wireless Network Security, Mobile Ad Hoc Networks and its routing issues, Intrusion Detection Systems and Security Design Issues. He is also a member of ACM GOLD Affinity Group, Professional Communication and Computer Society. Also, he is member of ACM and international Association of Engineers.

Dr. Nabeel Mohammed Zanoon, He received his $\mathrm{PhD}$ in Computer System Engineering, from South-West State University, Kursk, Russia, in 2011. He is faculty member with a AlBalqa' Applied University since 2011; where he is currently Assistant Professor and Head of Applied Sciences Department. Also, He is Director of the Computer Center and Cisco Academy with Aqaba University College. He has published several researches in several area, security of E-Banking, algorithm scheduling in Grid and Cloud, Meta-Grammar, Hardware and Architectural Computer, Fiber Optical and Mobile Ad Hoc Networks.

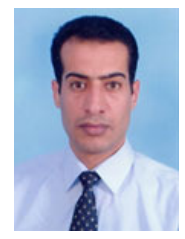


International Journal of Ad hoc, Sensor \& Ubiquitous Computing (IJASUC) Vol.7, No.2, April 2016

Rasha M. Al-Tarawneh received her B.Sc. and M.Sc. degree in Computer Science from Al-Balqa`Applied University, Salt, Jordan, in 2008 and 2011 respectively. Since 2011, she has been with the faculty of the Department of Applied Sciences at Al-Balqa' Applied University/Aqaba University College, Aqaba, Jordan; where she is currently a full time Lecturer.

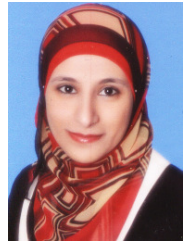

